SOZIALINFORMATIK

\title{
Was Führungskräfte wissen müssen
}

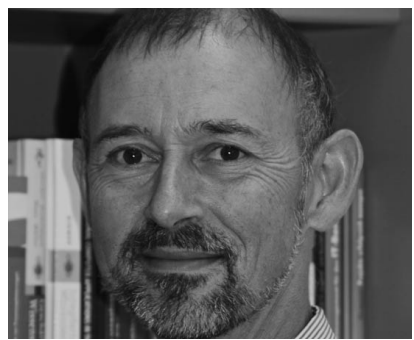

VON PROF. HELMUT KREIDENWEIS

Kreidenweis lehrt an der Katholischen Universität Eichstätt-Ingolstadt. Er ist dort Leiter des Masterstudiengangs Sozialinformatik und der Arbeitsstelle für Sozialinformatik. Zudem ist er Inhaber der IT-Beratung $\mathrm{KI}$ Consult in Augsburg. helmut.kreidenweis@kueichstaett.de
Auch soziale Unternehmen sind heute auf die Nutzung von Informationstechnologie existenziell angewiesen. Führungskräfte müssen deshalb erstens wissen, wie sie diese Möglichkeiten in ihrer Organisation einsetzen können und zweitens die Fähigkeit haben, den Einsatz von Informationstechnologie auf die Soziale Arbeit mit den Adressaten und die binnenorganisatorischen Prozesse zu analysieren.

Die Nutzung von Informationstechnologie (IT) in der Sozialwirtschaft ist Alltag, über die Verwaltung hinaus durchdringt sie zunehmend fachliche und steuernde Prozesse. Mehr als 60 Prozent der Mitarbeiter sozialer Organisationen arbeiten regelmäßig am Computer und jährlich investiert die Branche rund 200 Millionen Euro allein für fachspezifische Software (Kreidenweis/ Halfar, 2008). Die Techniknutzung ist breit gefächert: vom Rechnungswesen und Controlling über die Planung und Dokumentation von Hilfen bis hin zur direkten Adressatenarbeit in Form von Online-Beratung oder computerunterstütztem Lernen.

Blickt man auf die Aus- und Weiterbildungslandschaft, so spiegelt sich der praktische Stellenwert der Informationstechnologie dort nur gebrochen wider. Weder an den Fachschulen noch in den meisten Fachhochschul- und Universitätsstudiengängen für Soziale Arbeit oder in den weiterbildenden Masterstudiengängen für Sozialmanagement ist die Sozialinformatik fest im Fächerkanon verankert. In den rund 80 Fachhochschul-Studiengängen in Deutschland gibt es nur eine Handvoll ausgewiesener Sozialinformatik-Professuren. Ansonsten sind es oft an diesem Thema interessierte Lehrkräfte, die entsprechende Kurse anbieten.

Die Fachdiskussion zur sozialinformatischen Bildung wird heute keineswegs intensiver geführt als Ende des vergangenen Jahrhunderts (vgl. Kirchlechner/Kolleck 1991, Ohnemüller, 1996). In den letzten fünf Jahren sind lediglich drei dezidierte Fachbeiträge erschienen (Bredl/Halfar 2007, Kreiden- weis 2004, Mosebach/Göppner, 2005) und zu allem Überfluss wurde so mancher Diskurs auf den Punkt verkürzt, ob Programmieren nun zur Kernkompetenz von Sozialarbeiterinnen und Sozialarbeitern zählen soll (Jurgovsky 2004, Janatzek, 2007). Dies leitet unmittelbar zur zentralen Frage, weshalb sozialinformatisches Wissen in den genannten Ausbildungsgängen überhaupt vermittelt werden soll und welche Lehrinhalte sich für welche Bildungsgänge mit Blick auf die Zukunft als sinnvoll erweisen.

\section{Bildungsinhalte spezifisch vermitteln}

Die Antwort auf das Weshalb gliedert sich in eine instrumentelle und eine reflexive $\mathrm{Di}$ mension: Zum einen geht es darum, Handlungswissen zur Implementierung und zum nutzbringenden und sicheren Betrieb der Informationstechnologie zu vermitteln. Ziel ist hier ein effektiver und effizienter Einsatz in sozialen Organisationen. Zum anderen gilt es, die Fähigkeit zur kritischen Reflexion der intendierten Wirkungen und nicht intendierten Risiken und Nebenwirkungen des IT-Einsatzes auf die Arbeit mit den Adressaten und die binnenorganisatorischen Prozesse zu vermitteln. Die Frage nach den konkreten Ausbildungsinhalten kann nur auf dem Hintergrund der spezifischen Ausbildungsgänge und ihrer jeweiligen Zielperspektiven sinnvoll beantwortet werden.

Zunächst ist jedoch eine Verständigung darüber von Nöten, welche Wissensgebiete die Sozialinformatik umfasst und wo die Disziplin ihre Grenzpflöcke einschlägt bzw. auf Übergänge zu anderen Disziplinen verweist. Immer dann von Sozialinformatik zu sprechen, wenn »das Soziale « irgendwie mit dem Computer in Berührung kommt, scheint angesichts der heutigen Alltagsdurchdringung mit Informationstechnologie obsolet. Vielmehr muss der Fokus auf die arbeitsfeldspezifischen Formen der ITKonzeptualisierung, -Nutzung und -Evaluierung (vgl. Wendt 2000, S. 20) gerichtet 
werden. Sinnvolle disziplinäre Grenzen können sicherlich zur Medienpädagogik oder zur empirischen Sozialforschung gezogen werden: Auch dort werden Computer im sozialen Sektor angewandt, doch die Medienpädagogik präsentiert sich seit über 30 Jahren ein eigenständiges Wissensgebiet und die Forschung in der Sozialen Arbeit nutzt Software lediglich als Instrument für mathematische Operationen, genauso wie das die empirische Forschung etwa in der Soziologie oder Psychologie tut. Daher sollte sich die Sozialinformatik ebenso wie etwa die Verwaltungs-, Medizin- oder andere Fachinformatiken an den Kern-, Management- und Unterstützungsprozessen ihres Gegenstandsbereiches, hier also der Sozialen Arbeit und des Sozialmanagements und deren Umweltbezüge orientieren.

Grundlage jeder sozialinformatischen Ausbildung ist freilich die viel zitierte »computer literacy", also der souveräne Umgang mit der Kulturtechnik Computernutzung. Auch wenn die heutige Jugendgeneration oft als "computer natives « bezeichnet wird, für die soziale Netzwerke im Web 2.0 eine Sozialisationsinstanz darstellen und die Informatik an den Schulen Einzug gehalten hat, so gibt es noch immer bildungs- und milieuabhängige Unterschiede.
Auch kann die jugendspezifische Art der Technikaneignung nicht ohne weiteres mit Grundkenntnissen in Datenmanagement, Präsentationssoftware oder Tabellenkalkulation gleichgesetzt werden. Dennoch: Weiterführende Bildungsinstanzen sollten sich nur noch in Ausnahmefällen um IT-Basiskenntnisse kümmern müssen. Insbesondere mit Blick auf die oft verkürzten Ausbildungsgänge muss der Fokus klar auf dem fachlichen IT-Einsatz liegen.

\section{Spezialausbildung Sozialinformatik?}

Jenseits der Integration in die verschiedenen Bildungsgänge stellt sich die Frage, ob die Sozialwirtschaft heute spezialisierte Ausbildungsgänge in Sozialinformatik benötigt. Dazu muss einerseits das Tätigkeitsspektrum möglicher Absolventen ausgeleuchtet werden und andererseits gilt es zu sichten, inwieweit das benötigte Qualifikationsprofil nicht schon durch vorhandene Ausbildungsgänge abgedeckt ist.

Als primäres Einsatzgebiet für speziell ausgebildete Sozialinformatiker kann sicherlich die Position des IT-Verantwortlichen oder IT-Leiters in größeren Sozial- organisationen gelten. Hier ist die Zuständigkeit für sämtliche IT-Themen von der Hardware- und Netzwerk-Technik über Betriebssysteme und Office-Anwendungen bis hin zur fachspezifischen Software für Klientenverwaltung oder Dienstplanung sowie IT-Sicherheit verortet. Weitere Tätigkeitsfelder können in Beratung, Vertrieb und Projektleitung bei Fachsoftware-Anbietern oder im Bereich der anbieterunabhängigen IT-Beratung für soziale Organisationen gesehen werden. Zentrale Qualifikationen sind in all diesen Bereichen neben solidem IT-Wissen ein strategisch ausgerichtetes Denken, Management-Fähigkeiten sowie ein grundlegendes Verständnis der unterschiedlichen Prozesse, die es mit Informationstechnologie zu unterstützen gilt.

Die stark auf Systemtechnik oder Anwendungsentwicklung ausgerichtete klassische Informatik-Ausbildung bietet hierfür keine geeignete Grundlage. Weit eher werden Kompetenzen dieser Art von fachinformatischen Studiengängen wie Wirtschaftsoder Verwaltungsinformatik vermittelt. Dort fehlt jedoch das Wissen um die Spezifik sozialer Dienstleistungsprozesse, Organisationskontexte und Denkstrukturen, die den IT-Einsatz in der Sozialbranche ganz wesentlich determinieren.

\section{Sozialinformatik in der Bildungslandschaft}

Angesichts einer sich immer weiter ausdifferenzierenden Bildungslandschaft mit teils hoch spezialisierten Studiengängen und Ausbildungswegen kann kein detailliertes Konzept für eine Verankerung sozialinformatischer Bildungsinhalte geliefert werden. Vielmehr soll anhand dreier wesentlicher Ausbildungsebenen - Fachschule, Bachelorstudium der Sozialen Arbeit und (Weiterbildungs-) Masterstudium Sozialmanagement - eine beispielhafte Implementierung vorgestellt werden.

\section{- Fachschulen}

Als Schwerpunkt der Sozialinformatik-Ausbildung an Fachschulen oder Fachakademien kann die instrumentelle Ebene in Form einer exemplarischen Einführung in fachspezifische Software für die Planung und Dokumentation von Hilfen angesehen werden. Weitere Aspekte sind Datenschutz und IT-Sicherheit sowie auf der reflektorisch-kritischen Ebene die Frage nach dem »Datenschatten «, also digitalen Abbildung komplexer menschlicher Kontexte in IT-Systemen und deren Kongruenz mit der Realität.

\section{- Bachelor-Studium der Sozialen Arbeit}

Studierende der Sozialen Arbeit sollten darüber hinaus Fachsoftware auf dem Hintergrund fachlicher Konzepte wie etwa CaseManagement hinsichtlich ihrer Oualität und ihres Nutzens beurteilen können. Weitere Inhalte können die Themen Online-Beratung oder fachspezifische Nutzungsformen des Internets bilden. Wird eine Schwerpunktbildung in Sozialinformatik angeboten, so kann sich diese beispielsweise auf Teilaspekte des IT-Managements wie Fachsoftware-Auswahl oder fachliche Systemadministration, auf konzeptionelle Aspekte der Online-Beratung oder die fachliche und barrierefreie Konzeptionierung von Websites oder IT-Anwendungen für soziale Organisationen beziehen. Auf der reflektorischen Ebene kann das Thema »digitale Spaltung « mit Fokus auf die Adressaten Sozialer Arbeit vertieft behandelt werden.

\section{- Master-Studium Sozialmanagement}

Angesichts der geschilderten Bedeutung der Informationstechnologie für die verschiedenen Prozesse innerhalb sozialer Organisationen erscheint strategisches Wissen zur Steuerung der Informationstechnologie für Führungskräfte unverzichtbar. Dies reicht von Orientierungswissen zu Systemarchitekturen über die Abbildung von Geschäftsprozessen in Informationstechnologie bis zur Bildung und Nutzung IT-bezogener Kennzahlen oder Fragen des Outsourcings. Insbesondere in kleineren Organisationen, die sich keine eigene IT-Abteilung leisten können, müssen Führungskräfte darüber hinaus IT-Projekte wie die Auswahl und Einführung von Software-Systemen leiten können und für die Gewährleistung der IT-Sicherheit Sorge tragen. 
An der Katholischen Universität Eichstätt-Ingolstadt startete im Oktober 2009 daher erstmals in Deutschland ein zweijähriger berufsbegleitender Masterstudiengang Sozialinformatik. Die unterschiedlichen Ausgangsqualifikationen der 16 Studierenden werden dabei durch Wahlpflicht-Module in den Bereichen Soziale Arbeit, Betriebswirtschaft und Informatik abgefedert. Zentrale Lehrinhalte sind u. a. Grundlagen der angewandten Informatik, Geschäftsprozess-Management, Informationsmanagement, branchenspezifische IT-Lösungen, strategisches und operatives IT-Management sowie Netzwerke und IT-Sicherheit. Damit soll gleichermaßen strategische wie operatives Know-how für IT-Verantwortliche der Branche vermittelt werden.

\section{Fazit}

Die bisherige Diskussion um die Sozialinformatik in die Ausbildung war weitgehend auf klassischen Diplomstudiengänge an Fachhochschulen verkürzt, wobei die Position der einzelnen Autoren oft stärker mit ihrem spezifischen fachlichen Fokus auf die Basisdisziplin als mit den Anforderungen der beruflichen Praxis korrespondierte.

Hier wird dagegen für eine differenzierte Sichtweise plädiert: Auf der Basis der verschiedenen beruflichen Anforderungen an Fach- und Führungskräfte, die sich auch in der Grundkonzeption der einzelnen Ausbildungsgänge widerspiegeln, soll die Sozialinformatik in einer jeweils angemessenen Form implementiert werden. Der disziplinäre Streit darüber, was die Sozialinformatik nun ist oder nicht ist, rückt damit zugunsten einer qualifikationsorientierten Perspektive in den Hintergrund: Pädagogisch orientierte Ausbildungsgänge benötigen einen anderen Zugang zu diesem Fachgebiet als etwa managementorientierte Bildungsangebote oder Studienprogramme für IT-Verantwortliche.

Kernaufgabe der kommenden Jahre wird es sein, Curricula für alle Ausbildungsebenen (weiter) zu entwickeln und - mindestens ebenso wichtig - entsprechendes Lehrpersonal auszubilden. Um sich diesem Ziel zu nähern, ist ein Umdenken in den oberen Etagen der Wohlfahrtsverbände und Bildungsträger von Nöten: Informationstechnologie darf nicht länger auf rein technisches, an Spezialisten delegierbares Thema verengt werden. Weil sie in zunehmendem Maße Fach- und Führungsprozesse durchdringt, muss die Informationstechnologie von den Akteuren aller Ebenen verstanden, aktiv genutzt (mit-) gesteuert und kritisch reflektiert werden. Nicht weniger als dies, gilt es in der Ausbildung künftiger Fach- und Führungskräfte der Sozialwirtschaft zu vermitteln.

\section{Literatur}

Bredl, Klaus/Halfar, Bernd (2007): Lehrkonzepte für die Sozialinformatik: Welche Differenzierungen in der Lehre sind sinnvoll? in: Kreidenweis, Helmut/Halfar, Bernd (Hg.), Sozialinformatik Perspektiven für Praxis, IT-Entwicklung, Forschung und Lehre. Dokumentation der 2. Eichstätter Fachtagung, Eichstätt, 2007, S. 85-92. Janatzek, Uwe (2007): Sozialinformatik in der Sozialen Arbeit. Saarbrücken 2007.

Jurgovsky, Manfred (2004): Sozioinformatik. Ein Vorschlag zur Neupositionierung der Informatik in der Sozialen Arbeit, in: Archiv für Wissenschaft und Praxis der sozialen Arbeit, 1/2004, S. 40-48. Kirchlechner, Berndt (1987): Computereinsatz in Lehre, Forschung und Organisation an den Fachbereichen Sozialpädagogik und Sozialarbeit der Fachhochschule Frankfurt am Main, in: Frommann, Matthias (Hg.), Dezentrale Elektronische Datenverarbeitung in der sozialen Arbeit, Frankfurt am Main, 1987, S. 202-215. Kirchlechner, Berndt/Kolleck, Bernd 1991: Überlegungen zur EDV-Ausbildung in den Sozialwesenfachbereichen. In: Soziale Arbeit, Nr. 1, S. 18-22. Kreidenweis, Helmut (2004): Sozialinformatik in der Lehre. Ein Konzept zur systematischen Verankerung in der Ausbildung, in: Archiv für Wissenschaft und Praxis der sozialen Arbeit, 4/2004, S. 102-112.

Kreidenweis, Helmut/Halfar, Bernd (2008): IT-Report für die Sozialwirtschaft 2008/2009, Eichstätt 2008.

Ohnemüller, Bernhard 1996: Was müssen Profis künftig können? EDV-Ausbildung für die Soziale Arbeit. In: Kreidenweis, H. u. a. Hg.: EDV im Sozialwesen. Kongress-Dokumentation COSA ' 96. Freiburg im Breisgau.

Wendt, Wolf Rainer (Hg.): Sozialinformatik. Baden-Baden, 2000.

\section{Praxisgerechte Unterstützung}

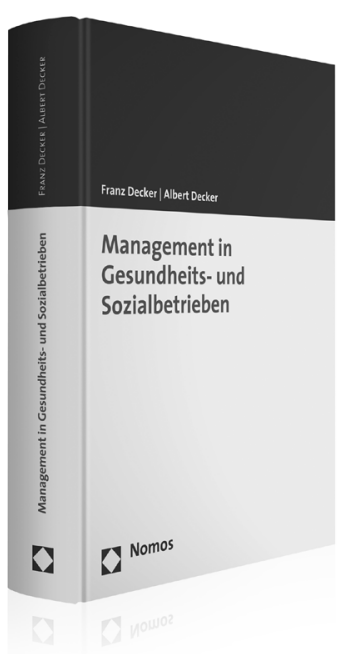

\section{Management in Gesund- heits- und Sozialbetrieben \\ Betriebswirtschaftliche Grundlagen für Führungskräfte und Nachwuchs}

Von em. Prof. Dr. rer. pol. Franz Decker und Dr. Albert Decker

2. Auflage 2008, 432 S., geb., 74,- $€$, ISBN 978-3-8329-2990-9

"Das Werk von Franz und Albert Decker bietet in einer Zeit der sich ständig ändernden Imponderabilien einen Leitfaden für ein effizienzorientiertes Verhalten und Handeln in Gesundheits- und Sozialbetrieben. Es gehört zu jenen Werken, die im Sozialmanagement unerlässlich erscheinen."

Prof. Dr. Dr. habil. Peter Eisenmann, www.socialnet.de September 2009

"Mit Beispielen und Schaubildern verständlich präsentiert, bietet dasHandbuch einen hervorragenden Grundriss. Es ist gleichermaßen für den Einstieg wie für die Vertiefung geeignet. Hier schreiben Praktiker, die wissen, worauf es ankommt...Insgesamt eine Empfehlung."

Ronald Richter, Altenheim 3/og

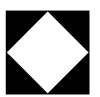

Nomos

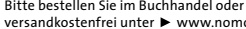

